\title{
Erikson's Theory of Psychosocial Development
}

\author{
Moin Syed \\ University of Minnesota \\ Kate C. McLean \\ Western Washington University
}

To appear in: E. Braaten \& B. Willoughby (Eds.), The SAGE Encyclopedia of Intellectual and Developmental Disorders

Contact: moin@umn.edu or kate.mclean@wwu.edu 
Erik Erikson's theory of psychosocial development is the first, and arguably most influential, lifespan theory of development. Erikson's writings are extensive and complicated, covering quite a bit of conceptual ground. He mixed detailed treatments with vague proclamations, and returned to the same themes repeatedly throughout his career. These qualities of his work have led some to refer to his work as having "Rorschach-like" qualities, where different readers glean and interpret his words based on their own interests and views. Thus, it is a fool's task to attempt to represent Erikson in full or to detail the "true" nature of Erikson's theory. Accordingly, this article contains a description of the primary thematic elements of the theory.

Erikson was highly influenced by Freud's psychoanalytic theory of development, but extended it in two substantial ways. First, Freud's focus was limited to childhood, arguing that the bulk of personality is formed around age five (following the phallic stage). In contrast, Erikson developed a lifespan theory; that is, he theorized about the nature of personality development as it unfolds from birth through old age. Second, Freud's theory is considered a psychosexual theory of development, emphasizing the importance of sexual drives and genitalia in how children develop. Erikson's theory is considered psychosocial, emphasizing the importance of social and cultural factors across the lifespan. Despite Erikson's departure from the sexual primacy of Freud's theorizing, Erikson's theory is undoubtedly a psychoanalytic theory strongly influenced by Freud. Yet, although social tensions are highlighted above sexual tensions, Erikson' theory still accords a prominent role for infantile sexuality, lifelong libidinal drives, and the unconscious. Thus, Erikson's theory is quite a bit more expansive than Freud's. It accounts for a greater range of psychological domains (e.g., sexual and social) as well as a much larger chunk of the lifespan (i.e., all of it).

The most well-known aspect of Erikson's theory is his description of eight fundamental psychosocial tensions that individuals must balance throughout their lives (Figure 1). For this reason, we focus on this aspect of his theory in this chapter. The eight tensions are frequently referred to as "stages." Using the stage concept to characterize the tensions, however, has led to widespread misunderstanding of a critical aspect of Erikson's theory. Thinking of the tensions as stages can lead to a belief that the tensions occur in a particular sequence, not becoming relevant until their anointed time, and no longer being of concern once the tension has been "resolved." This is far from the case. Rather, Erikson argued that all eight of the tensions are present within individuals at all points in the lifespan, but that specific tensions are salient at different points in time. Thus, a specific tension will manifest prior to its primary developmental period, and will continue to be relevant after. Figure 1 provides a visual representation of this feature of Erikson's theory with the use of continuous arrows.

Tension five, identity vs. role confusion, provides a clear example of this continuity. Erikson suggested that the period of adolescence and young adulthood was the primary developmental period to resolve the identity tension. However, identity-related issues can certainly arise prior to adolescence, which is supported by extensive research on mirror self-recognition, self-other integration, and role preferences; issues that arise from infancy 
through childhood. Likewise, individuals' identities are not achieved, completed, or unchanging following young adulthood. Both normative (e.g., marriage, children) and nonnormative (e.g., divorce, job-loss) events can lead to continued identity negotiation in adulthood. In general, from an Eriksonian perspective, no aspect of development is ever actually "complete."

In modern terms, the eight psychosocial tensions can be thought of as developmental tasks. Developmental tasks are prevalent, if not universal, psychological issues that individuals must confront and resolve in order to realize healthy development. Thinking of the eight tensions as developmental tasks helps us understand the psychological issues that are particularly salient to individuals at different points in their lives.

A question that often comes up is the role of age in Erikson's theory. As mentioned earlier, a key feature of the theory is that it covers the lifespan. Because it is a lifespan theory, consisting of eight age-graded tensions, there is often a desire to assign specific age ranges to each of the tensions. However, this is not possible. There are no hard and fast ages at which each tension rises to prominence and falls to the background. This is true for three main reasons. First, as mentioned, all eight tensions are present at all points in development. Second, there are individual differences in the length and intensity with which individuals engage in each tension. For some, the identity tension is particularly challenging and long-lasting, while for others it is the intimacy tension that is prolonged. Third, despite widespread belief, Erikson paid close attention to the cultural context of the life-course, particularly among marginalized groups in the U.S. (e.g., women, African Americans, Native Americans), and how cultural factors modulated the life course.

Thus, how long individuals seriously engage with each tension depends on their own personal situations and cultural contexts. That said, there are general age periods in which each tension rises to prominence, and we describe these below. Moreover, the tensions have a sequential quality in that the reasonable resolution of a tension is necessary to successfully resolve a subsequent tension. To put it another way, unresolved tensions earlier in life can lead to challenges down the road. We will provide examples of this process, too.

Trust vs. Mistrust (infancy). The basic and fundamental psychological task is for infants to develop a sense that their caregivers and environments are responsive, reliable, and consistent. In other words, that they can trust that their basic needs will be met. Basic trust is facilitated by a responsive caregiver. When infants are in distress-whether it be due to hunger, exhaustion, injury, or a dirty diaper-can they expect that a caregiver will attend to their needs? This developmental task has been extensively elaborated in theory and research on attachment, most notably by John Bowlby and Mary Ainsworth.

Autonomy vs. Shame and Doubt (toddlerhood). Following infants' understanding of an predictable environment, that they can depend on others, toddlerhood is characterized by the initial step towards developing as an individual person; that is, developing a sense of autonomy. As with all Eriksonian tensions, the manifestation of this task is associated with contemporary biological and cultural circumstances. Accordingly, the primary route 
through which toddlers begin to establish a sense of autonomy is through defecation and the process of toilet training. In a world in which toddlers have very little control, this is one domain in which they both have some control and are expected to exercise it. Erikson discussed toilet training as the beginnings of understanding the processes of "holding on and letting go," which are fundamental to future development.

Initiative vs. Guilt (early childhood). The third tension takes autonomy one step further. In early childhood, children learn to take greater risks in separating from their caregiver, actively engaging with their environments on their own terms-taking initiative. This most often takes the form of independent play, with children demonstrating the ability to engage with concrete materials or their own imaginations. The negative pole, guilt, corresponds to the guilt associated with engaging in work that is not intricately tied to the caregiver-that initiating independent play serves as a betrayal of the established bond.

Industry vs. Inferiority (middle childhood). The fourth tension is a direct extension of the previous. Indeed, although all eight of the tensions form a successive developmental sequence, tensions 2-4 represent a clear sequence in the development of autonomy. Building upon the ability to take initiative, industry is the ability to see projects through to completion. It is characterized by having the interest, skills, and motivation to persist at a task, and is related to gains in self-regulation. Importantly, this is the prime period for developing proficiency in culturally-specific technologies. What it means to "create" will vary due to particular cultural demands and expectations, and successful resolution of this task involves a mastery of these technologies.

Identity vs. Role Confusion (adolescence and emerging adulthood). At the dawn of adolescence, individuals begin to engage in a new and special kind of project: themselves. Identity was a central concept in Erikson's thinking, and his writings have made a huge impact on subsequent theory and research, as well as popular culture. Indeed, Erikson was the first to write about, and use the term, "identity crisis." Although this term is part of the everyday vernacular in many parts of the world, this was far from the case prior to Erikson.

Intimacy vs. Isolation (emerging adulthood and adulthood). As youth move even deeper into adulthood, developing intimate relationships becomes particular salient. Importantly, intimacy here involves both romantic and platonic relations-it is about sharing oneself with others. Indeed, once individuals develop a reasonable sense of identity, they are then prepared to share that identity with others in order to develop successful intimate relations. Ideally, when two individuals develop an intimate relationship, they establish an identity for the relationship while each maintaining his/her own individual identities. Here we can see a clear example of how the tensions build upon each other. If an individual who does not have a clear sense of identity attempts to engage in a relationship, there is a danger of that person defining oneself in terms of the relationship, with no individual identity defined apart from it. If that relationship endsand it likely will-it can have destructive effects because the individual has little to fall back on. 
Generativity vs. Stagnation (adulthood). Once individuals move into adulthood proper, they begin to think about what they want to leave behind. That is, now that they have developed an identity and shared it with other people, what of themselves do they want to pass on to future generations? This is the thrust of generativity. For many, generativity takes the form of having children, as reproduction contributes to survival of our species and allows individuals to transmit their culture. However, reproduction is not the only expression of generativity. Engaging in activities that will benefit future generations, including mentoring, teaching, artistic ventures, civic participation, and activism, are all ways in which people can develop a sense of generativity; that they have something worth passing along to subsequent generations.

Integrity vs. Despair (old age). In old age, the primary developmental task becomes a reflective one. Do people feel like, when they look back on their life, that they did things the right way? That they lived the best life that they good? Or are they filled with regrets, asking themselves whether they could have made different choices that led to better opportunities. This is the essence of the final tension. It is has a markedly different feel than the other seven tensions, as its primary function is evaluative vs. productive. That is, it is more about being than it is about doing.

As a parting point, it is important to understand that the concept of balance is central to Erikson's theory. Each of the eight tensions consists of two opposing poles, a "positive" end and a "negative" end. When individuals are engaged in the developmental task associated with each tension, their primary psychological work involves finding a sense of balance between these two poles. They will be pulled towards both the positive and negative poles during this time, and the goal is to arrive at a feeling of successful resolution of the positive and negative elements. In our descriptions above we emphasized the positive poles, but the ideal state is to retain some aspects of the negative pole while realizing most of the positive. Take Basic Trust: it is not considered adaptive to be all-trusting, but rather some degree of mistrust can serve individuals well in their lives. In sum, there is a great deal of complexity in Erikson's theory, and we invite the interested reader to engage with some of the suggested reading to learn more about it.

\section{Suggested Readings}

Erikson, E. H. (1950). Childhood and society. New York: W W Norton \& Co.

This is the most complete articulation of Erikson's theory, and a good starting point for those interested in engaging in his ideas. Although some find his prose to be terse and thus difficult to digest, there is no substitute for the original source material if one is truly interested in Erikson's complex ideas.

Erikson, E. H. (1968). Identity: Youth and crisis. New York: Norton.

This book is a collection of Erikson's essays on the subject of identity, which ultimately was his greatest scholarly focus and undoubtedly his greatest contribution to psychology. 
Friedman, L. J. (2000). Identity's architect: A biography of Erik H. Erikson. Cambridge, MA: Harvard University Press.

The definitive biography of Erikson and his life’s work.

McLean, K. C., \& Syed, M. (Eds.). (2015). The Oxford handbook of identity development. New York: Oxford University Press.

Containing 34 original chapters, this handbook represents the state-of-the-field of contemporary theory and research on Erikson's theory of identity development and related approaches. 


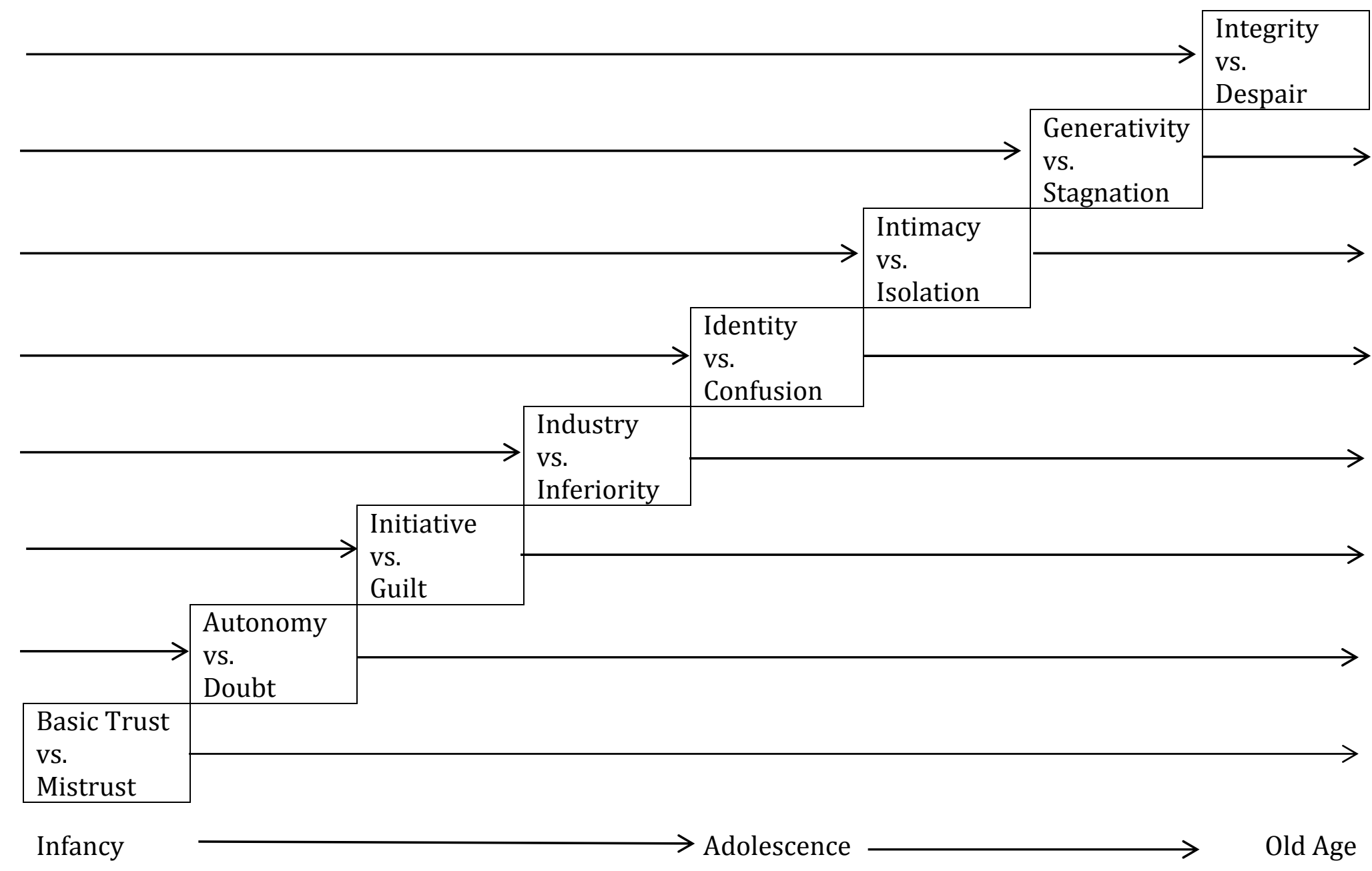

Figure 1. Depiction of Erikson's eight psychological tensions, highlighting how all tensions are present at all points in development, but that certain tensions are salient at particular development periods in the lifespan 